\title{
THE WIGNER DISTRIBUTION FUNCTION FOR SYSTEMS OF BOSONS OR FERMIONS
}

\author{
by $\mathrm{K}$. SCHRAM and B. R. A. NIJBOER \\ Instituut voor theoretische fysica, Rijksuniversiteit te Utrecht, Nederland
}

\section{Synopsis}

An expression for the Wigner distribution function valid for systems of bosons or fermions is obtained by making use of correspondence relations between classical quantities and quantum mechanical operators first given by Groenewold. A general and straightforward derivation of the equation of motion for the Wigner distribution function is presented. The equation governing the temperature dependence of the Wigner distribution function in the case of a canonical ensemble can be derived in a completely analogous way.

$\S 1$. Introduction. In quantum statistics a mixed state is usually described by the density operator $\rho$, which in Dirac's notation is defined as

$$
\rho=\Sigma_{\mu} p_{\mu}|\mu\rangle\langle\mu|
$$

where $p_{\mu}$ is the statistical weight of the pure normalized state $|\mu\rangle$ (the ensemble of pure states $|\mu\rangle$ is not necessarily orthogonal or complete). If $p_{\mu}$ is normalized according to $\Sigma_{\mu} p_{\mu}=1$ then, by virtue of the definition (1), the ensemble average of an operator $\mathscr{A}$ can be written as

$$
\langle\mathscr{A}\rangle=\Sigma_{\mu} p_{\mu}\langle\mu|\mathscr{A}| \mu\rangle=\operatorname{Tr}(\mathscr{A} \rho)
$$

In the literature $\rho$ is also often defined by its matrix elements $\rho_{k l}=$ $=\langle k|\rho| l\rangle$ where $|k\rangle$ and $|l\rangle$ belong to some complete orthonormal set in Hilbert space. Especially the coordinate representation proves to be quite useful. In this representation the density matrix $\rho\left(\boldsymbol{r}^{\prime}, \boldsymbol{r}^{\prime \prime}\right)$ can be expressed in terms of an arbitrary complete orthonormal set of wavefunctions $\varphi_{f}(\boldsymbol{r})$ as

$$
\rho\left(\boldsymbol{r}^{\prime}, \boldsymbol{r}^{\prime \prime}\right)=\Sigma_{\jmath} \varphi_{j}^{*}\left(\boldsymbol{r}^{\prime \prime}\right) \rho \varphi_{j}\left(\boldsymbol{r}^{\prime}\right)
$$

The equation of motion for $\rho$ has in the Schrödinger representation the well-known form

$$
\frac{\partial \rho}{\partial t}=-i \hbar^{-1}[\mathscr{H}, \rho]
$$

where $\mathscr{H}$ is the hamiltonian and $[\mathscr{H}, \rho]$ the commutator $\mathscr{H}_{\rho}-\rho \mathscr{H}$. In the 
special case of statistical equilibrium described by a canonical ensemble the (unnormalized) density operator is given by

$$
\rho=\exp (-\beta \mathscr{H}), \beta=(k T)^{-1}
$$

It is seen to satisfy the so-called Bloch equation

$$
\frac{\partial \rho}{\partial \beta}=-\mathscr{H}_{\rho}
$$

An alternative formulation of quantum statistics by means of a distribution function in phase space, proposed by Wigner ${ }^{1}$ ), has been studied by several authors ${ }^{2-5}$ ). In this formalism a mixed state of a system consisting of $N$ particles is represented by a distribution function $f\left(\boldsymbol{r}_{1} \ldots \boldsymbol{r}_{N}\right.$, $\left.\boldsymbol{p}_{1} \ldots \boldsymbol{p}_{N}\right)$ of the coordinates $\boldsymbol{r}_{\boldsymbol{i}}$ and the canonically conjugate momenta $\boldsymbol{p}_{i}$, in such a way that the quantum mechanical ensemble average of an arbitrary operator $\mathscr{A}$ can be calculated according to

$\langle\mathscr{A}\rangle=\int a\left(\boldsymbol{r}_{1}, \ldots, \boldsymbol{r}_{N}, \boldsymbol{p}_{1}, \ldots, \boldsymbol{p}_{N}\right) f\left(\boldsymbol{r}_{1}, \ldots, \boldsymbol{r}_{N}, \boldsymbol{p}_{1}, \ldots, \boldsymbol{p}_{N}\right) \mathrm{d} \boldsymbol{r}_{1} \ldots \mathrm{d} \boldsymbol{r}_{N} \mathrm{~d} \boldsymbol{p}_{1} \ldots \mathrm{d} \boldsymbol{p}_{N}(7)$

where $a\left(r_{1} \ldots r_{N}, p_{1} \ldots p_{N}\right)$ represents the classical quantity corresponding to the quantum mechanical operator $\mathscr{A}$. If, in particular, the correspondence between $a$ and $\mathscr{A}$ is established according to Weyl's rule (cf. $\S 2$ ), one finds for $f\left(\boldsymbol{r}_{1} \ldots \boldsymbol{r}_{N}, \boldsymbol{p}_{1} \ldots \boldsymbol{p}_{N}\right)$ the expression $\left.{ }^{\boldsymbol{b}}\right)$

$$
f(\boldsymbol{r}, \boldsymbol{p})=(\pi \hbar)^{-3 N} \int \rho(\boldsymbol{r}-\boldsymbol{y}, \boldsymbol{r}+\boldsymbol{y}) \exp \left(2 i \hbar^{-1} \boldsymbol{p} \cdot \boldsymbol{y}\right) \mathrm{d} \boldsymbol{y}
$$

(for convenience, the vectors $\boldsymbol{r}_{1} \ldots \boldsymbol{r}_{N}$ and $\boldsymbol{p}_{\mathbf{1}} \ldots \boldsymbol{p}_{N}$ will be indicated by $3 N$-dimensional vectors $\boldsymbol{r}$ and $\boldsymbol{p}$ throughout this paper; further all integrations are to be extended from $-\infty$ to $+\infty)$. The function $f(\boldsymbol{r}, \boldsymbol{p})$ given by (8) is real, but not necessarily non-negative. It is called the Wigner distribution function (W.d.f.).

Both formulae (7) and (8) are valid for systems of identical particles with Maxwell-Boltzmann statistics as well as for systems of bosons or fermions. In the definition (1) of $\rho$ the state vector $|\mu\rangle$ should then of course have the proper symmetry with respect to permutations of the particles. The same applies to the complete system of wave functions $\varphi_{j}(\boldsymbol{r})$ in (3). In order to investigate the influence of the statistics on the W.d.f. somewhat more closely, it turns out to be more convenient to use another representation of $f(\boldsymbol{r}, \boldsymbol{p})$, namely a representation in terms of a complete orthogonal set of functions $f_{k l}(\boldsymbol{r}, \boldsymbol{p})$ rather than the Fourier integral formula (8). These functions $f_{k l}(\boldsymbol{r}, \boldsymbol{p})$ will be introduced in the next section, where also a brief survey of important correspondence relations will be given.

§2. Correspondence relations between operators and functions of $r, p$. Many years ago Weyl ${ }^{7,8}$ ) proposed the following prescription for constructing a hermitian operator $\mathscr{A}$ from a classical quantity $a(\boldsymbol{r}, \boldsymbol{p})$ which is 
a real function of the coordinates and momenta: one expands $a\left(r_{\mathbf{b}} \boldsymbol{p}\right)$ in a Fourier integral according to

$$
a(\boldsymbol{r}, \boldsymbol{p})=\int \alpha(\boldsymbol{\sigma}, \boldsymbol{\tau}) \exp \{i(\boldsymbol{\sigma} . \boldsymbol{r}+\boldsymbol{\tau} \cdot \boldsymbol{p})\} \mathrm{d} \boldsymbol{\sigma} \mathrm{d} \boldsymbol{\tau}
$$

The operator $\mathscr{A}$ corresponding to $a(\boldsymbol{r}, \boldsymbol{p})$ is then obtained from $(9 a)$ by replacing $\boldsymbol{r}$ and $\boldsymbol{p}$ by the corresponding operators $\mathscr{R}$ and $\mathscr{P}$ (usually one takes $\mathscr{R}=\boldsymbol{r}, \mathscr{P}=-i \hbar \nabla)$ :

$$
\mathscr{A}(\mathscr{R}, \mathscr{P})=\int \alpha(\boldsymbol{\sigma}, \boldsymbol{\tau}) \exp \{i(\boldsymbol{\sigma} . \mathscr{R}+\tau . \mathscr{P})\} \mathrm{d} \boldsymbol{\sigma} \mathrm{d} \boldsymbol{\tau}
$$

By means of this rule a $(1,1)$-correspondence is established between hermitian operators $\mathscr{A}$ and real functions $a(\boldsymbol{r}, \boldsymbol{p})$.

Now, if $|k\rangle$ and $|l\rangle$ belong to some complete orthonormal set in Hilbert space, the matrixelenent $A_{k l}=\langle k|\mathscr{A}| l\rangle$ can obviously be written as $A_{k l}=\int \alpha(\boldsymbol{\sigma}, \boldsymbol{\tau})\langle k|\exp \{i(\boldsymbol{\sigma} . \mathscr{R}+\boldsymbol{\tau} . \mathscr{P})\}| l\rangle \mathrm{d} \boldsymbol{\sigma} \mathrm{d} \boldsymbol{\tau}=$ $=(2 \pi)^{-\boldsymbol{\sigma} N} \int a(\boldsymbol{r}, \boldsymbol{p}) \exp \{-i(\boldsymbol{\sigma} . \boldsymbol{r}+\boldsymbol{\tau} \cdot \boldsymbol{p})\}\langle k|\exp \{i(\boldsymbol{\sigma} . \mathscr{R}+\boldsymbol{\tau} . \mathscr{P})\}| l\rangle \mathrm{d} \boldsymbol{\sigma} \mathrm{d} \boldsymbol{\tau} \mathrm{d} \boldsymbol{r} \mathrm{d}=$ where

$$
=\int a(\boldsymbol{r}, \boldsymbol{p}) f_{k l}(\boldsymbol{r}, \boldsymbol{p}) \mathrm{d} \boldsymbol{r} \mathrm{d} \boldsymbol{p} \quad(10 a)
$$

$f_{k l}(\boldsymbol{r}, \boldsymbol{p})=(2 \pi)^{-6 N} \int \exp \{-i(\boldsymbol{\sigma} . \boldsymbol{r}+\boldsymbol{\tau} \cdot \boldsymbol{p})\}\langle k|\exp \{i(\boldsymbol{\sigma} . \mathscr{R}+\boldsymbol{\tau} . \mathscr{P})\}| l\rangle \mathrm{d} \boldsymbol{\sigma} \mathrm{d} \boldsymbol{\tau}(11)$

The functions $f_{k l}(\boldsymbol{r}, \boldsymbol{p})$ were introduced by Groenewold $\left.{ }^{2}\right)$ and independently by Moyal ${ }^{3}$ ) who proved for the case of one degree of freedom that they form a complete orthogonal set. Hence we also have

$$
a(\boldsymbol{r}, \boldsymbol{p})=h^{3 N} \sum_{k l} A_{k l} f_{l k}(\boldsymbol{r}, \boldsymbol{p})
$$

Moreover, these complex functions have the important property to correspond in the sense of Weyl to the non-hermitian operators $h^{-3 N}|l\rangle\langle k|$

$$
h^{-3 N}|l\rangle\langle k| \leftrightarrow f_{k l}(\boldsymbol{r}, \boldsymbol{p})
$$

a correspondence which has been demonstrated by Groenewold ${ }^{2}$ ).

Let now $w(\boldsymbol{r}, \boldsymbol{p})$ be the function which corresponds according to Weyl's prescription to the density operator $h^{-3 N} \rho$

$$
h^{-3 N} \rho \leftrightarrow w(\boldsymbol{r}, \boldsymbol{p})
$$

then, as follows from (1) and (12)

$$
h^{-3 N} \rho=h^{-3 N} \Sigma_{\mu} p_{\mu}|\mu\rangle\langle\mu| \leftrightarrow w(\boldsymbol{r}, \boldsymbol{p})=\Sigma_{\mu} p_{\mu} f_{\mu \mu}(\boldsymbol{r}, \boldsymbol{p})
$$

(the arrow $\leftrightarrow$ always means Weyl's correspondence). Furthermore, since an arbitrary operator $\mathscr{A}$ can be expanded into

$$
\mathscr{A}=\sum_{k l} A_{k l}|k\rangle\langle l|
$$

one finds from (12) and (13) taking $\mathscr{A}=h^{-3 N} \rho$

$$
h^{-3 N} \rho=h^{-3 N} \sum_{k l} \rho_{k l}|k\rangle\langle l| \leftrightarrow w(\boldsymbol{r}, \boldsymbol{p})=\Sigma_{k l} \rho_{k l} f_{l k}(\boldsymbol{r}, \boldsymbol{p})
$$


The righthand side of (16) is seen to be a special case of (10b), with $\mathscr{A}=$ $=h^{-3 N} \rho, a(\boldsymbol{r}, \boldsymbol{p})=w(\boldsymbol{r}, \boldsymbol{p})$. As a consequence of $(10 a)$ and $(16)$ the ensemble average of an arbitrary operator $\mathscr{A}$ can be written as

$$
\begin{aligned}
& \langle\mathscr{A}\rangle=\operatorname{Tr}(\mathscr{A} \rho)=\Sigma_{k l} A_{l k} \rho_{k l}= \\
& \quad=\Sigma_{k l} \int a(\boldsymbol{r}, \boldsymbol{p}) f_{l k}(\boldsymbol{r}, \boldsymbol{p}) \rho_{k l} \mathrm{~d} \boldsymbol{r} \mathrm{d} \boldsymbol{p}=\int a(\boldsymbol{r}, \boldsymbol{p}) w(\boldsymbol{r}, \boldsymbol{p}) \mathrm{d} \boldsymbol{r} \mathrm{d} \boldsymbol{p}
\end{aligned}
$$

Comparing (17) to (7) we conclude that

$$
f(\boldsymbol{r}, \boldsymbol{p}) \equiv w(\boldsymbol{r}, \boldsymbol{p})
$$

i.e. the W.d.f., defined by (7), turns out to be the function which according to Weyl's rule corresponds to the density operator $h^{-3 N} \rho$.

So far no symmetry conditions have been imposed on the wave functions. It is easily verified, however, that the form of all the foregoing formulae does not change when the state vectors $|\mu\rangle$ and the basis $|k\rangle,|l\rangle$ are restricted to the Hilbert space of symmetric or antisymmetric functions. If the density operator belonging to an ensemble of (anti)symmetric states $|\mu\rangle$ is denoted by $\rho^{ \pm}$and the function which according to Weyl's rule corresponds to this operator by $f^{ \pm}(\boldsymbol{r}, \boldsymbol{p}),(17)$ can be immediately rewritten as

$$
\begin{aligned}
& \langle\mathscr{A}\rangle=\operatorname{Tr}\left(\mathscr{A} \rho^{ \pm}\right)=\Sigma_{k l}^{\prime} A_{l k} \rho_{k l}= \\
& \quad=\Sigma_{k l}^{\prime} \int a(\boldsymbol{r}, \boldsymbol{p}) f_{l k}(\boldsymbol{r}, \boldsymbol{p}) \rho_{k l} \mathrm{~d} \boldsymbol{r} \mathrm{d} \boldsymbol{p}=\int a(\boldsymbol{r}, \boldsymbol{p}) f^{ \pm}(\boldsymbol{r}, \boldsymbol{p}) \mathrm{d} \boldsymbol{r} \mathrm{d} \boldsymbol{p}
\end{aligned}
$$

where $\Sigma^{\prime}$ denotes a restricted summation over a complete set of either symmetric or antisymmetric states and where

$$
f^{ \pm}(\boldsymbol{r}, \boldsymbol{p})=\Sigma_{k l}^{\prime} \rho_{k l} f_{l k}(\boldsymbol{r}, \boldsymbol{p})
$$

a relation which is completely analogous to (16).

Hence $f^{ \pm}(\boldsymbol{r}, \boldsymbol{p})$ is the W.d.f. for a system of bosons or fermions. It should be stressed here, that in general $f^{ \pm}(\boldsymbol{r}, \boldsymbol{p})$ is not the same function as $f(\boldsymbol{r}, \boldsymbol{p})$ and similarly $\rho^{ \pm}$is not the same operator as $\rho$. This becomes clear when we observe that it is always possible to construct in Hilbert space a set of basisvectors $|k\rangle$ in such a way that the (anti)symmetric subspace is spanned by a subset $\left|k^{\prime}\right\rangle$ belonging to $|k\rangle$. Whereas in (16) the summation indices $k$ and $l$ are extended over a complete set in the whole of Hilbert space, in (20) the summation should be restricted to the subspace of (anti)symmetric functions. For example, in the case of a canonical ensemble the density operator $\rho=\exp (-\beta \mathscr{H})$ operates on the whole of Hilbert space and has matrix elements between arbitrary states $|k\rangle$ and $|l\rangle$, whereas the elements of the matrix of $\rho^{ \pm}$vanish if $|k\rangle$ and $|l\rangle$ are orthogonal to the (anti)symmetric subspace.

The function $f(\boldsymbol{r}, \boldsymbol{p})=w(\boldsymbol{r}, \boldsymbol{p})$ can be found, at least in principle, from $\rho$ by applying Weyl's rule in the form of (9) or (16) and similarly $f \pm(r, p)$ can be 
obtained from $p^{ \pm}$by means of (9) or (20). However, it would appeąr to be useful to express $f^{ \pm}$in the more familiar function $f(\boldsymbol{r}, \boldsymbol{p})$. Now it turns out that $(20)$ can easily be transformed into a relation between $f^{ \pm}$and $f$, a relation which has previously been established by J. Vlieger ${ }^{9}$ ) in a different manner. To derive this relation the following properties of the functions $f_{k l}(\boldsymbol{r}, \boldsymbol{p})$ are needed:

$$
f_{k l}(\boldsymbol{r}, \boldsymbol{p})=f_{l k} *(\boldsymbol{r}, \boldsymbol{p})
$$

$\Sigma_{k l}^{\prime} f_{k l}(\boldsymbol{r}, \boldsymbol{p}) f_{k l} *^{*}\left(\boldsymbol{r}^{\prime}, \boldsymbol{p}^{\prime}\right)=\left(\frac{2}{h}\right)^{6 N} \frac{1}{N !} \Sigma_{\mathrm{P}}( \pm)^{\mathrm{P}} \int \delta\left(\mathrm{Pr}-\mathrm{P} \boldsymbol{x}-\boldsymbol{r}^{\prime}-x^{\prime}\right) \times$

$$
\times \delta\left(\boldsymbol{r}^{\prime}-\boldsymbol{x}^{\prime}-\boldsymbol{r}-\boldsymbol{x}\right) \exp \left\{-2 i \hbar^{-1}\left(\boldsymbol{p} \cdot \boldsymbol{x}+\boldsymbol{p}^{\prime} \cdot \boldsymbol{x}^{\prime}\right)\right\} \mathrm{d} \boldsymbol{x} \mathrm{d} \boldsymbol{x}^{\prime}
$$

$\delta(\boldsymbol{r})$ is a shorthand notation for the product $\delta\left(\boldsymbol{r}_{1}\right) \delta\left(\boldsymbol{r}_{2}\right) \ldots \delta\left(\boldsymbol{r}_{N}\right), \mathrm{P}$ is an operator which permutes $\boldsymbol{r}_{1}, \boldsymbol{r}_{2}, \ldots \boldsymbol{r}_{N} \cdot(+)^{\mathbf{P}}=1,(-)^{\mathbf{P}}=+$ for even, - for odd permutations. The $(+)$ sign always refers to $B-E$ statistics, the (-) sign to F-D statistics.

The relation (21) follows directly from the definition (11), (22), however, is less trivial. As a matter of fact, it.is a generalization of a completeness relation derived by Moyal ${ }^{3}$ ) for the case of one degree of freedom. It is easily obtained if one uses a representation of $f_{k l}(\boldsymbol{r}, \boldsymbol{p})$ in terms of an orthonormal set of (anti)symmetric wave functions $\varphi_{k}(\boldsymbol{r})$,

$$
f_{k l}(\boldsymbol{r}, \boldsymbol{p})=(2 / h)^{3 N} \int \varphi_{k}^{*}(\boldsymbol{r}-\boldsymbol{x}) \varphi_{l}(\boldsymbol{r}+\boldsymbol{x}) \exp \left(-2 i \hbar^{-1} \boldsymbol{p} \cdot \boldsymbol{x}\right) \mathrm{d} \boldsymbol{x}
$$

which follows from (11) after some obvious transformations, and if one bears in mind that the wave functions $\varphi_{k}(\boldsymbol{r})$ satisfy the completeness relation

$$
\Sigma_{k}^{\prime} \varphi_{k}(\boldsymbol{r}) \varphi_{k}^{*}\left(\boldsymbol{r}^{\prime}\right)=(1 / N !) \Sigma_{\mathbf{P}}( \pm)^{\mathbf{P}} \boldsymbol{\delta}\left(\mathrm{P} \boldsymbol{r}-\boldsymbol{r}^{\prime}\right)
$$

because they form an orthonormal basis in the (anti)symmetric subspace of Hilbert space. Using (10a), (21) and (22) (with $\mathscr{A}=h^{-3 N} \rho$ ) one finds starting from (20)

$$
\begin{aligned}
f^{ \pm}(\boldsymbol{r}, \boldsymbol{p}) & =\Sigma_{k l}^{\prime} \rho_{k l} f_{l k}(\boldsymbol{r}, \boldsymbol{p})=h^{3 N} \sum_{k l}^{\prime} \int w\left(\boldsymbol{r}^{\prime} \boldsymbol{p}^{\prime}\right) f_{k l}\left(\boldsymbol{r}^{\prime}, \boldsymbol{p}^{\prime}\right) f_{k l} *^{*}(\boldsymbol{r}, \boldsymbol{p}) \mathrm{d} \boldsymbol{r}^{\prime} \mathrm{d} \boldsymbol{p}^{\prime}= \\
= & (4 / h)^{3 N}(1 / N !) \Sigma_{\mathbf{P}}( \pm)^{\mathbf{P}} \int w\left(\boldsymbol{r}^{\prime}, \boldsymbol{p}^{\prime}\right) \delta\left(\mathrm{P} \boldsymbol{r}-\mathrm{P} \boldsymbol{x}-\boldsymbol{r}^{\prime}-\boldsymbol{x}^{\prime}\right) \times \\
& \times \delta\left(\boldsymbol{r}^{\prime}-\boldsymbol{x}^{\prime}-\boldsymbol{r}-\boldsymbol{x}\right) \exp \left\{-2 i \hbar^{-1}\left(\boldsymbol{p} . \boldsymbol{x}+\boldsymbol{p}^{\prime} \cdot \boldsymbol{x}^{\prime}\right) \mathrm{d} \boldsymbol{r}^{\prime} \mathrm{d} \boldsymbol{p}^{\prime} \mathrm{d} \boldsymbol{x} \mathrm{d} \boldsymbol{x}^{\prime}\right.
\end{aligned}
$$

By means of this relation, which as was mentioned above was first derived by Vlieger ${ }^{9}$ ) in a somewhat different way, the W.d.f. for a system of bosons or fermions can be obtained directly from the W.d.f. for the corresponding system obeying Maxwell-Boltzmann statistics. If, for example, (25) is applied to the case of an ideal gas, one obtains the correct expression for the Slatersum for an ideal quantum gas, as will be shown in the appendix. Incidentally we may remark that $f(\boldsymbol{r}, \boldsymbol{p})$ for a system in statistical equilibrium at not too low temperatures can be approximated by a series expansion 
in $\hbar^{2}$, which has already been shown by Wigner ${ }^{1}$ ). On substituting this series into (25) one finds an approximate expression for $f^{ \pm}(\boldsymbol{r}, \boldsymbol{p})$. The resulting expression, however, is not a simple expansion into powers of $\hbar$ but is of a more complicated nature.

The advantage of introducing the operator $\rho^{ \pm}$, which is not strictly needed for the derivation of (25), will become apparent in the next section.

§ 3. Dependence on time and temperature. The W.d.f. has been used on the one hand in the quantum theory of transport phenomena ${ }^{10}$ ), on the other hand in calculations of quantum corrections for systems in thermodynamic equilibrium ${ }^{1,11}$ ). In transport theory one needs the equation of motion for the W.d.f., giving the way in which it depends on time. It is always obtained from (8) by differentiation with respect to time (cf. $\left.{ }^{10}\right)$ where references to earlier literature are given). In the case where the hamiltonian is given by

$$
\mathscr{H}=-\frac{\hbar^{2}}{2 m} \Delta+V(\boldsymbol{r})
$$

one finds by substituting (4) and by partial integration and series expansion of $V(\boldsymbol{r})$

$$
\frac{\partial f}{\partial t}+\frac{1}{m} \boldsymbol{p} \cdot \frac{\partial f}{\partial \boldsymbol{r}}-\frac{2}{\hbar} \sin \left(\frac{\hbar}{2} \frac{\partial}{\partial \boldsymbol{p}} \frac{\partial}{\partial \boldsymbol{r}}\right) f(\boldsymbol{r}, \boldsymbol{p}, t) V(\boldsymbol{r})=0
$$

where $\partial / \partial \boldsymbol{r}$ in the third term operates on $V(\boldsymbol{r})$ only.

- In the calculations of quantum corrections the dependence of $f(\boldsymbol{r}, \boldsymbol{p}, \beta)$ on temperature is of interest. In a paper by Oppenheim and Ross ${ }^{11}$ ) it is derived in a way analogous to that of the equation of motion discussed above. Instead of (4) the Bloch equation (6) is used; for a hamiltonian of the form (26) one is, after rather lengthy calculations, led to

$$
\frac{\partial f}{\partial \beta}-\left(\frac{\hbar^{2}}{8 m} \Delta-\frac{\boldsymbol{p}^{2}}{2 m}\right) f(\boldsymbol{r}, \boldsymbol{p}, \beta)-\cos \left(\frac{\hbar}{2} \frac{\partial}{\partial \boldsymbol{p}} \frac{\partial}{\partial \boldsymbol{r}}\right) f(\boldsymbol{r}, \boldsymbol{p}, \beta) V(\boldsymbol{r})=0
$$

where $\partial / \partial \boldsymbol{r}$ in the last term again operates on $V(\boldsymbol{r})$ only.

In this section we want to draw attention to the fact that both equations (27) and (28) (and generalizations thereof) can be obtained directly from(4) and (6) respectively by making use of the following rules:

(i) Weyl's correspondence between $\rho$ and $f(\boldsymbol{r}, \boldsymbol{p})$ or between $\rho^{ \pm}$and $f^{ \pm}(\boldsymbol{r}, \boldsymbol{p})$, as discussed in $\S 2$.

(ii) If $\mathscr{A} \leftrightarrow a(\boldsymbol{r}, \boldsymbol{p})$ and $\mathscr{B} \leftrightarrow b(\boldsymbol{r}, \boldsymbol{p})$ according to Weyl's prescription, then

$$
\begin{aligned}
& \frac{1}{2} i[\mathscr{A}, \mathscr{B}]= \\
& \quad=\frac{1}{2} i(\mathscr{A} \mathscr{B}-\mathscr{B} \mathscr{A}) \leftrightarrow \sin \frac{\hbar}{2}\left(\frac{\partial}{\partial \boldsymbol{p}_{a}} \frac{\partial}{\partial \boldsymbol{r}_{b}}-\frac{\partial}{\partial \boldsymbol{r}_{a}} \frac{\partial}{\partial \boldsymbol{p}_{b}}\right) a(\boldsymbol{r}, \boldsymbol{p}) b(\boldsymbol{r}, \boldsymbol{p})
\end{aligned}
$$




$$
\begin{aligned}
& \frac{1}{2}\{\mathscr{A}, \mathscr{B}\}= \\
& \quad=\frac{1}{2}(\mathscr{A} \mathscr{B}+\mathscr{B} \mathscr{A}) \leftrightarrow \cos \frac{\hbar}{2}\left(\frac{\partial}{\partial \boldsymbol{p}_{a}} \frac{\partial}{\partial \boldsymbol{r}_{b}}-\frac{\partial}{\partial \boldsymbol{r}_{a}} \frac{\partial}{\partial \boldsymbol{p}_{b}}\right) a(\boldsymbol{r}, \boldsymbol{p}) b(\boldsymbol{r}, \boldsymbol{p})
\end{aligned}
$$

where $\partial / \partial \boldsymbol{p}_{a}$ means $\partial / \partial \boldsymbol{p}$ operating on $a(\boldsymbol{r}, \boldsymbol{p})$ only, etc. The rules of correspondence $(28 a, b)$ between classical quantities and the (anti)commutator of their corresponding quantum mechanical operators, which do not seem to be generally known, were first derived by Groenewold $\left.{ }^{2}\right)$; (28a) was established independently by Moyal $\mathbf{3}$ ).

Now, putting $\mathscr{A}=\mathscr{H} \leftrightarrow H(\boldsymbol{r}, \boldsymbol{p}), \mathscr{B}=h^{-3 N} \rho$ and applying. (i) and (ii) to the equation of motion (4) for $\rho$, one finds immediately

$$
\frac{\partial f}{\partial t}=-\frac{2}{\hbar} \sin \frac{\hbar}{2}\left(\frac{\partial}{\partial \boldsymbol{p}_{H}} \frac{\partial}{\partial \boldsymbol{r}_{f}}-\frac{\partial}{\partial \boldsymbol{r}_{H}} \frac{\partial}{\partial \boldsymbol{p}_{f}}\right) H(\boldsymbol{r}, \boldsymbol{p}) f(\boldsymbol{r}, \boldsymbol{p}, t)
$$

In the special case

$$
H=\frac{1}{2} \boldsymbol{p}^{2} / m+V(\boldsymbol{r})
$$

(29) reduces to (27) again, but (29) could be applied as well to systems of charged particles in an electromagnetic field for example. The general form (29) of the equation of motion for the W.d.f. has been derived in a different way by Moyal ${ }^{3}$ ) in the case of one degree of freedom. Evidently (29) is independent of the statistics, because one might have put equally well $\mathscr{B}=h^{-3 N} \rho^{ \pm}$in stead of $\mathscr{B}=h^{-3 N} \rho$. Hence $f^{ \pm}$obeys the same equation of motion, and the effect of the statistics only appears in the initial condition for (29).

In a similar way one can proceed to derive the equation for the temperature dependence of the W.d.f. in the case of a canonical ensemble. As the density operator (5) in this case commutes with the hamiltonian, the Bloch equation can be written in the form

$$
\frac{\partial \rho}{\partial \beta}=-\frac{1}{2}\{\mathscr{H}, \rho\}
$$

Applying again (i) and (ii) one obtains

$$
\frac{\partial f}{\partial \beta}=-\cos \frac{\hbar}{2}\left(\frac{\partial}{\partial \boldsymbol{p}_{H}} \frac{\partial}{\partial \boldsymbol{r}_{f}}-\frac{\partial}{\partial \boldsymbol{r}_{H}} \frac{\partial}{\partial \boldsymbol{p}_{f}}\right) H(\boldsymbol{r}, \boldsymbol{p}) f(\boldsymbol{r}, \boldsymbol{p}, \beta)
$$

If the hamiltonfunction takes the special form (30), equation (32) immediately reduces to (28). As far as we are aware, however, the generalized form (32) of this equation has not been published before. Furthermore it should be realized that, because (31) holds also for $\rho^{ \pm},(32)$ again, just as (29), is independent of the statistics. i.e. that (32) is equally valid for $f^{ \pm}$.

As a conclusion we wish to thank Prof. Dr. H. J. Groen e wold, Groningen and Mr. J. Vlieger, Leiden, for the useful discussions we had with them on this subject. 


\section{APPENDIX}

Slatersum of an ideal quantum gas. The Slatersum $S(\boldsymbol{r})$ which for a canonical ensemble is defined as the diagonal element $\rho(\boldsymbol{r}, \boldsymbol{r})$ of the density matrix in the coordinate representation, can be written as (cf. (8))

$$
S(\boldsymbol{r})=\rho(\boldsymbol{r}, \boldsymbol{r})=\int f(\boldsymbol{r}, \boldsymbol{p}) \mathrm{d} \boldsymbol{p}
$$

or, for a system of bosons and fermions as

$$
S \pm(\boldsymbol{r})=\int f^{ \pm}(\boldsymbol{r}, \boldsymbol{p}) \mathrm{d} \boldsymbol{p}
$$

In the case of an ideal quantum gas $(V(\boldsymbol{r})=0)$ the expression (5) for the density operator reduces to

$$
\rho=\exp \left(\frac{\beta \hbar^{2}}{2 m} \Delta\right)
$$

The corresponding classical function $w(\boldsymbol{r}, \boldsymbol{p})$ becomes simply

$$
w(\boldsymbol{r}, \boldsymbol{p})=h^{-3 N} \exp \left(-\frac{\beta}{2 m} \boldsymbol{p}^{2}\right)
$$

because $\rho$ is a function of the momentum operators only. Substituting (A.4) into (25) one obtains the following expression for the W.d.f. of an ideal quantum gas

$$
\begin{array}{r}
f^{ \pm}(\boldsymbol{r}, \boldsymbol{p}, \beta)=\left(\frac{2}{h}\right)^{6 N} \frac{1}{N !} \sum_{\mathrm{P}}( \pm)^{\mathrm{P}} \int \exp \left(-\frac{\beta}{2 m} \boldsymbol{p}^{2}\right) \delta\left(\mathrm{Pr}-\mathrm{P} \boldsymbol{x}-\boldsymbol{r}^{\prime}-\boldsymbol{x}^{\prime}\right) \times \\
\times \delta\left(\boldsymbol{r}^{\prime}-\boldsymbol{x}^{\prime}-\boldsymbol{r}-\boldsymbol{x}\right) \exp \left\{-2 i \hbar^{-1}\left(\boldsymbol{p} \cdot \boldsymbol{x}+\boldsymbol{p}^{\prime} \cdot \boldsymbol{x}^{\prime}\right) \mathrm{d} \boldsymbol{r}^{\prime} \mathrm{d} \boldsymbol{p}^{\prime} \mathrm{d} \boldsymbol{x} \mathrm{d} \boldsymbol{x}^{\prime}=\right. \\
=\left(\frac{1}{2}\right)^{3 N}\left(\frac{2}{h}\right)^{6 N}\left(\frac{2 \pi m}{\beta}\right)^{3 N / 2} \frac{1}{N !} \Sigma_{\mathrm{P}}( \pm)^{\mathrm{P}} \int \exp \left(-2 i \hbar^{-1} \boldsymbol{p} \cdot \boldsymbol{x}\right) \times \\
\quad \times \exp \left\{-\frac{1}{2}\left(m / \beta \hbar^{2}\right) \sum_{k=1}^{N}\left(\mathrm{P} \boldsymbol{r}_{k}-\mathrm{P} \boldsymbol{x}_{k}-\boldsymbol{r}_{k}-\boldsymbol{x}_{k}\right)^{2}\right\} \mathrm{d} \boldsymbol{x}
\end{array}
$$

(in the last line the index $k$ numbers the individual particles)

Integration over the momenta yields

$$
S \pm(\boldsymbol{r})=\frac{1}{N !} \lambda^{-3 N} \sum_{\mathbf{P}}( \pm)^{\mathbf{P}} \exp \left\{-\frac{\pi}{\lambda^{2}} \sum_{k=1}^{N}\left(\mathbf{P} \boldsymbol{r}_{k}-\boldsymbol{r}_{k}\right)^{2}\right\}
$$

with

$$
\lambda=h(2 \pi m k T)^{-\mathrm{i}}
$$

which is indeed the well-known expression ${ }^{12}$ ) for the Slatersum of an ideal quantum gas. 


\section{REFERENCES}

1) Wigner, E., Phys. Rev, 40 (1932) 749.

2) Groenewold, H. J. Physica 12 (1946) 405.

3) Moyal, J. E., Proc. Cambr. Phil. Soc. 45 (1949) 99.

4) Takabayasi, T., Progr. Theor. Phys. 11 (1954) 341

5) Baker, G. A., Phys. Rev, 109 (1958) 2198.

6) See e.g. Irving, J. H. and $Z$ wanzig, R. W., J. chem. Phys. 18 (1951) 1173.

7) Weyl, H., Z. Phys. 46 (1927) 1.

Gruppentheorie und Quantenmechanik, S. Hirzel, Leipzig $2^{\circ}$ Aufl. (1931) p. 244.

8) Shewell, J. R., Am. J. Phys. 27 (1959) 16.

9) Vlieger, J., personal communication.

10) Mori, H. and Ross, J., Phys. Rev. 109 (1958) 1877.

11) Oppenheim, I. and Ross, J., Phys. Rev. 107 (1957) 28.

12) See e.g. Kahn, B., Thesis, Utrecht 1938, p. 20. 